\title{
Outflowing Components in the Prototype Narrow-Line Seyfert 1 Galaxy Markarian 478
}

\author{
Qirong Yuan \\ Physics Department, Nanjing Normal Univ., Nanjing 210097, China \\ Micheal Brotherton \\ Department of Physics and Astronomy, University of Wyoming, \\ Laramie, WY 82071, USA \\ Richard F. Green \\ National Optical Astronomy Observatory, Tucson, AZ 85726-6732, USA \\ Gerard A. Kriss \\ Space Telescope Science Institute, Baltimore, MD 21218, USA
}

\begin{abstract}
Markarian 478 is the prototype narrow-line Seyfert 1 galaxy. Based on its spectra taken by the FUSE, HST/FOS, and Kitt Peak Observatory $2 \mathrm{~m}$ Telescope, we find a tendency that the emission lines of increasing ionization level show increasing excess flux at their blue wings and an increasing line peak velocity shift. This may indicate an outflowing component in the Broad-Line Region (BLR) where the ionization level increases with ejection velocity. In the mean time, the associated absorption lines are present on the blue wings of the high-ionization emission doublet O VI $\lambda \lambda 1032,1038$ and Lyman $\beta$ line. The physical condition of some absorbing components can be well constrained with the assistance of photoionization models. The flux ratios at the blue wings of emission lines of various ionization levels, as a function of outflow velocity, could also put important constraints on the physical condition of the outflowing components.
\end{abstract}

\section{Introduction}

Recent X-ray observations suggest that the narrow line Seyfert 1 galaxies (NLS1) have smaller black holes than normal Seyfert 1 galaxies and emit X-ray radiation with a higher efficiency (Hayashida 2000). Therefore, the NLS1 galaxies should be more likely to have outflowing gas, and associated and intrinsic absorption can be expected to appear in their UV and X-ray spectra with a higher rate. This paper mainly reports the discovery of outflowing components in the prototype NLS1 galaxy Markarian 478 (Mrk 478). 


\section{Spectral Imprint of Outflowing Gas}

Our analysis is based on far-UV, UV, and optical spectra of Mrk 478 which were obtained by the FUSE, the FOS/HST, and the $2 \mathrm{~m}$ telescope of Kitt Peak Observatory, respectively. The broad emission lines of O VI, C III, and N III in the FUSE spectrum are readily apparent.

The trend of increasing blueshift with increasing ionization level can be found in most quasars (e.g., Corbin 1990), but the typical blueshift of the highionization lines, such as C IV and $\mathrm{N} \mathrm{V}$, is only $\sim 200-300 \mathrm{~km} \mathrm{~s}^{-1}$. We also find this trend in Mrk 478. The high-ionization emission lines in our spectra are found to have very high blueshift velocities, about 4 times larger than found in a typical quasar. The highest blueshift, $-1444 \mathrm{~km} \mathrm{~s}^{-1}$, is displayed by the highest ionization doublet $\mathrm{O}$ VI, and it also exhibits the broadest profile. The higher ionization lines are all blueshifted by more than $300 \mathrm{~km} \mathrm{~s}^{-1}$, while the lower ionization lines blueshifted by $<300 \mathrm{~km} \mathrm{~s}^{-1}$. This trend may be produced by the outflowing gas in the broad-line region (BLR), where the ionization level increases with outflow velocity.

The narrow emission-line profiles in Mrk 478 and the high resolution of this far-UV spectrum minimize blending effects remarkably, which makes possible a significantly more accurate measurement of individual emission lines and the intrinsic absorption components superposed in the O VI doublet, C III, N III and $\mathrm{Ly} \beta$. We have identified five distinct kinematic components in O VI absorption lines, spanning a range of velocity from -2300 to $370 \mathrm{~km} \mathrm{~s}^{-1}$ relative to the systemic redshift of Mrk 478. For Ly $\beta$ absorption features, only three components are found. However, no associated absorption lines are detected in N III and C III emission profiles. The column density and covering factor for each absorption component can be then derived. With the assistance of photoionization models, the column density ratio $N_{O V I} / N_{H I}$ for some components will put important constraints on ionization parameters and total hydrogen column densities for the far-UV absorbing gas.

The outflowing gas in the BLR with the highest velocity may produce the associated UV absorption system (Laor et al. 1997). Modeling of the underlying power-law continuum and broad emission components is in process. A quantitative analysis on emission lines and absorption features, including the derivation of physical conditions of far-UV absorbers, will be presented in a separate paper.

Acknowledgments. QY wants to acknowledge the National Key Base Sciences Research Foundation under contract TG1999075402 and the Chinese NSF No. 10273007.

\section{References}

Corbin, M.R. 1990, ApJ, 357, 346

Hayashida, K. 2000, New Astro. Rev., 44, 419

Laor, A., et al. 1997, ApJ, 489, 656 